\title{
Animal-to-Animal Data Sharing Mechanism for Wildlife Monitoring in Fukushima Exclusion Zone
}

\author{
Hill Hiroki Kobayashi ${ }^{1, *}$, Keijiro Nakagawa ${ }^{2}$, Ko Makiyama ${ }^{2}$, Yuta Sasaki ${ }^{1}$, Hiromi Kudo ${ }^{1}$, \\ Baburam Niraula ${ }^{1}$ and Kaoru Sezaki ${ }^{1}$ \\ 1 Center for Spatial Information Science, The University of Tokyo, Tokyo 113-8656, Japan; \\ sasaki@csis.u-tokyo.ac.jp (Y.S.); kudo3@csis.u-tokyo.ac.jp (H.K.); bniraula@csis.u-tokyo.ac.jp (B.N.); \\ sezaki@csis.u-tokyo.ac.jp (K.S.) \\ 2 Department of Socio-Cultural Environmental Studies, The Graduate School of Frontier Science, \\ The University of Tokyo, Tokyo 113-8656, Japan; kenakaga@csis.u-tokyo.ac.jp (K.N.); \\ komakiyama@mcl.iis.u-tokyo.ac.jp (K.M.) \\ * Correspondence: kobayashi@csis.u-tokyo.ac.jp; Tel.: +81-4-7136-4291
}

Received: 20 April 2018; Accepted: 22 June 2018; Published: 3 July 2018

\begin{abstract}
We propose an animal-to-animal data sharing mechanism that employs wildlife-borne sensing devices to expand the size of monitoring areas in which electricity, information, and road infrastructures are either limited or nonexistent. With the proposed approach, monitoring information can be collected from remote areas in a safe and cost-effective manner. To substantially prolong the life of a sensor node, the proposed mechanism activates the communication capabilities only when there is a plurality of animals; otherwise, the sensor node remains in a sleep state. This study aimed to achieve three objectives. First, we intend to obtain knowledge based on the actual field operations within the Fukushima exclusion zone. Second, we attempt to realize an objective evaluation of the power supply and work base that is required to properly evaluate the proposed mechanism. Third, we intend to acquire data to support wildlife research, which is the objective of both our present (and future) research.
\end{abstract}

Keywords: sensor networks; nature conservation; wearable interfaces

\section{Introduction}

Wildlife monitoring via ubiquitous sensor networks and mobile phones has been realized in locations close to urban areas [1-3]; however, power and information infrastructural networks are severely limited in wildlife habitats because infrastructural services are not profitable in areas where the number of users is extremely small. Implementing a ubiquitous sensor network in wildlife habitats requires significant coordination with national parks, land owners, and administrative and other stakeholders. Additionally, installing sensors outdoors incurs enormous installation, operational, and environmental costs. Given these limitations, the wearable sensors for wildlife are attracting increasing attention.

However, while targeting wild animals, the total sensor weight is limited to less than $2 \%$ [4] of the animal's body weight, and there are few opportunities to regularly collect and charge the sensors. Whether this $2 \%$ limit is appropriate is an animal welfare or ethical question; however, because we are undertaking wearable sensor research, we cannot ignore the mass of the sensor. Therefore, in this study, sensors having a weight of less than this limit will be allowed with the permission of a veterinarian.) Further, to obtain the acquired sensor information, the animal must contact a sink node (a base station used to collect and process data) that is connected to an external network; however, the frequency of such contact is often low. Therefore, the design of collection methods for information obtained by low-power, high-reliability sensors in all environments is crucial. 
To address this problem, we propose an animal-to-animal data sharing system [5], as depicted in Figure 1. Using a multidisciplinary approach, we propose an animal behavior-based design and interfaces to connect animals and computer through a visually likable reaction (it is not an absolutely tied to brain wave measurement but is an action that seems likely to be due only to visual judgment). In a previous study (1996-2010), we used an animal-to-animal sharing system to monitor an endangered species on Iriomote Island, Japan [6]. Additionally, a prototype system was evaluated using laboratory animals (dogs) supervised by veterinarians [4]. The present study conducted an evaluation using cows in a more natural environment in a high radiation-dose area. The main contribution of this study is to clarify the observations that are relative to designing algorithms while targeting the animals in a natural environment. Adopting these earlier methods, we fabricated a wearable animal-to-animal data sharing device, constructed an our prototype, and conducted an on-site evaluation of cows in the Fukushima exclusion zone. In the following sections, we provide a conceptual overview of animal-to-animal interaction, discuss animal-wearable sensors for monitoring animal territories and animal-to-animal data sharing, and present an evaluation of the implementation within the Fukushima exclusion zone.

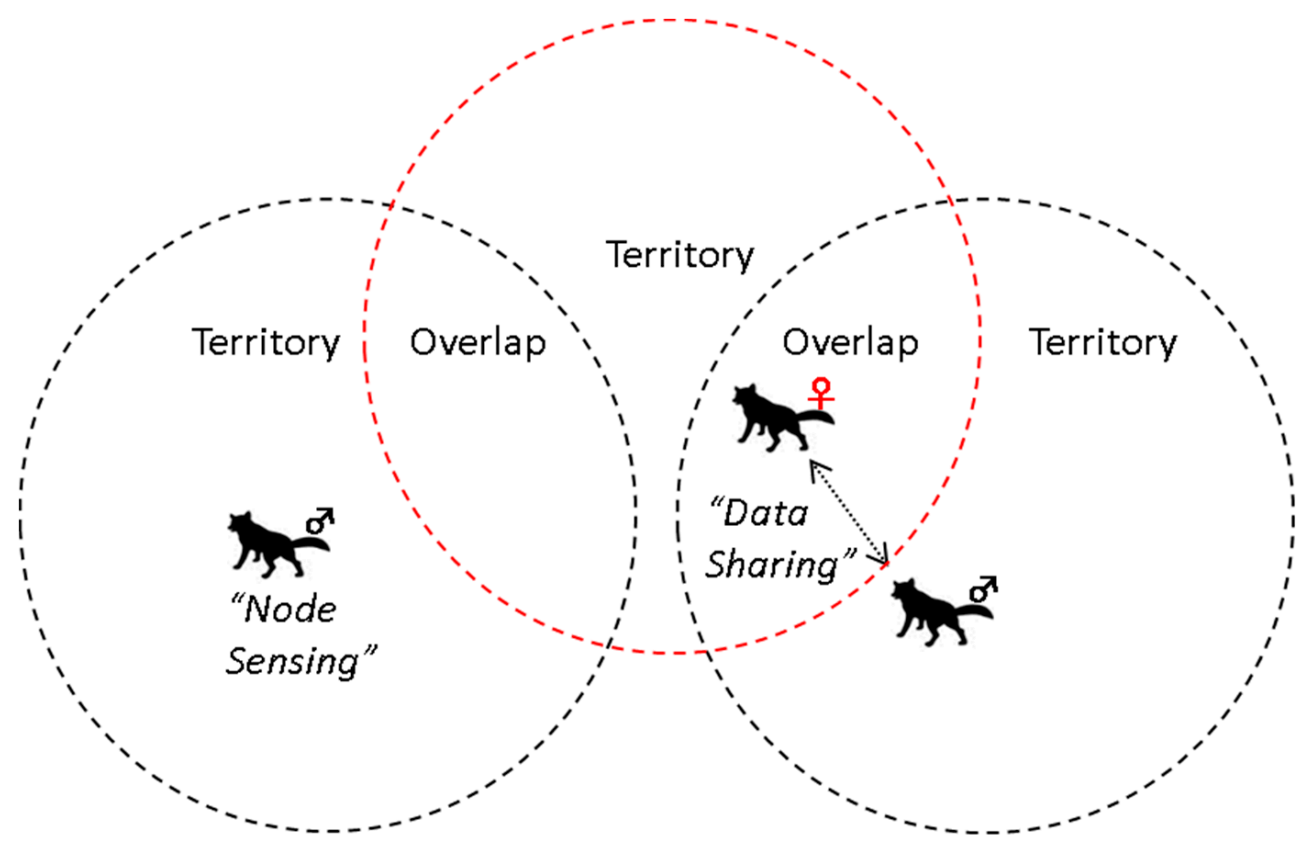

Figure 1. Adjacent and overlapping territories due to gender differences. By observing the behaviors of various territorial animals, wildlife wearing a type $q$ sensor near a sink node exhibits a high probability for encountering adjacent wildlife that wears a type $\sigma^{x}$ sensor. While encountering another individual, there is a high probability that both $q$ and $\sigma^{7}$ sensor nodes will be within the communication radius. By exploiting such territorial structure and gender differences, we propose that the observation range of wildlife wearing sensors can be expanded.

\section{Related Work and Problem Statement}

The concept that humans and animals will wear sensors to monitor the behavior and the surrounding environment can be observed even during the early stages of sensor network research. However, while targeting wild animals, there are limited opportunities to recharge the sensor batteries, and sensor weight is limited to be less than $2 \%$ of an animal's body weight. Additionally, to obtain the acquired sensor information, an animal must contact a sink node that eventually connects with an external network; however, the frequency of such contact is low. Therefore, low-power sensor nodes are essential. 
Takahashi of Fukushima University used wild monkeys to investigate the radioactive contamination caused by the Fukushima nuclear power plant accident [7]. Takahashi's experiments involved a dosimeter and a collar fitted with a Global Positioning System (GPS). To collect the radiation levels and position information, a sensor device ( $350 \mathrm{~g})$ was fitted to a captured female monkey's neck. The monkey was then released into the forest. Takahashi considered that a wearable sensor could obtain the distribution of radiation within a certain area. To increase the sensor operation time, Takahashi considered the social nature of monkeys, i.e., they live in groups (troops). Typically, females do not leave the group and have a high survival rate; therefore, Takahashi selected a female monkey. The sensor automatically disengaged while transmitting a signal. After two weeks, the instrument was collected, and the recorded data were analyzed. To obtain the sensor data, the female monkeymust be recaptured, which can be problematic. In addition, the monkey's habitat was limited; thus, the scope of observations was limited. To retrieve the acquired records via sensors, they must recapture wild animals or use a wearable telemetry transmitter. The habitat within which wild animals can be recaptured and the telecommunication range for telemetry are both extremely limited, which makes expanding the scope of surveys and studies difficult.

Given the above conditions, to conduct extensive and long-term wildlife surveys in habitats where power and information infrastructures are limited, we require a system that minimizes cost and maximizes efficiency. Here, two key problems must be resolved simultaneously.

1. Coverage: Wearable sensors are restricted to the physically recapturable individuals.

2. Operation time: Wearable sensors are restricted in terms of their operable time because the weight of the wearable device is limited to $2 \%$ of the animal's body weight.

From an engineering perspective, it was not possible to satisfy all the aforementioned specifications during a single development stage. Therefore, we conducted two subprojects. First, we focused on developing animal-to-animal sharing targeting dogs in our laboratory. Second, based on the first developments, we created a concept demonstration of animal-to-animal within the exclusion zone. Combining these two aspects, we evaluated animal-to-animal data sharing and its potential to enhance the capabilities of animal-wearable technologies.

\section{Animal-to-Animal Data Sharing Mechanism}

If a sensor can be attached to an individual animal, we can regularly sense and identify the corresponding area without requiring a mechanism to move the sensors. In many cases, animals are solitary and only group when mating or parenting. If we attach a sensor to solitary animals, we can only obtain the location and health information under such isolated conditions. If we attach a sensor to a mate or a child, the sensors may be able to communicate when they are in sufficiently close proximity.

Social animals interact in a group; however, the distance between individuals can vary over time. Therefore, a sensor attached to a specific individual can acquire position and health information. In addition, if all individuals in a group are equipped with sensors, inter-sensor communication is possible.

However, the weight of wearable batteries is limited for both solitary and social animals. Therefore, low-power sensors are required. In addition, the communication range is short. Therefore, the method to detect the approach of an animal wearing such a sensor is a significant problem. Generally, there is no alternative but to receive the signal of another party to verify whether there is a communicable sensor within range. If we can receive the signal of another party, we can confirm that there is another animal that wears a sensor within a communicable range. In contrast, if no signal can be received, no other animals with sensors are within the communicable range. However, to determine whether communication is possible, the communication system must be powered at all times, and, with sensors attached to wildlife, this is infeasible due the weight limitation. Thus, it is necessary to realize as much power saving as possible while targeting animals that cannot be easily recaptured. 
For a wireless sensor node, communication between sensors requires 100 times more power than that required by other operations [7]. When animals encounter a different individual, the probability that the sensor nodes on the given animals are within the necessary communication range is high. Therefore, by activating the communication capabilities of a sensor node only in the presence of multiple animals (otherwise, the sensor node is put into a sleep state), it is possible to substantially prolong the lifetime of the sensor node. To detect whether multiple animals are in proximity/communication range, this study uses ecological interactions among the species in animal communities [8] with an objective to perform a task-specific activity. Specifically, we demonstrate animal behaviors by classifying them into three steps (Figure 2). General terrestrial mammals, such as cats or dogs, exhibit a visually confirmed behavior that can be referred to as a pause and move while facing a detected object [3,9]. For example, if you meet another dog while walking your own dog, your dog pauses and then performs an action, such as barking, based on its interest in the other dog. Given the prevalence of such behavior, we opted to measure the three steps by obtaining synthetic acceleration data. However, this cannot measure and confirm brain waves. We consider the behavior to be an action likely to move the device. Based on the amount of interest shown by the animals, we can determine specific behaviors from synthetic three-dimensional acceleration data, thereby distinguishing between the target (multiple animals) and non-target conditions and behaviors. Thus, we implemented this behavior-oriented trigger to initiate data exchange for any application involving wild animals.

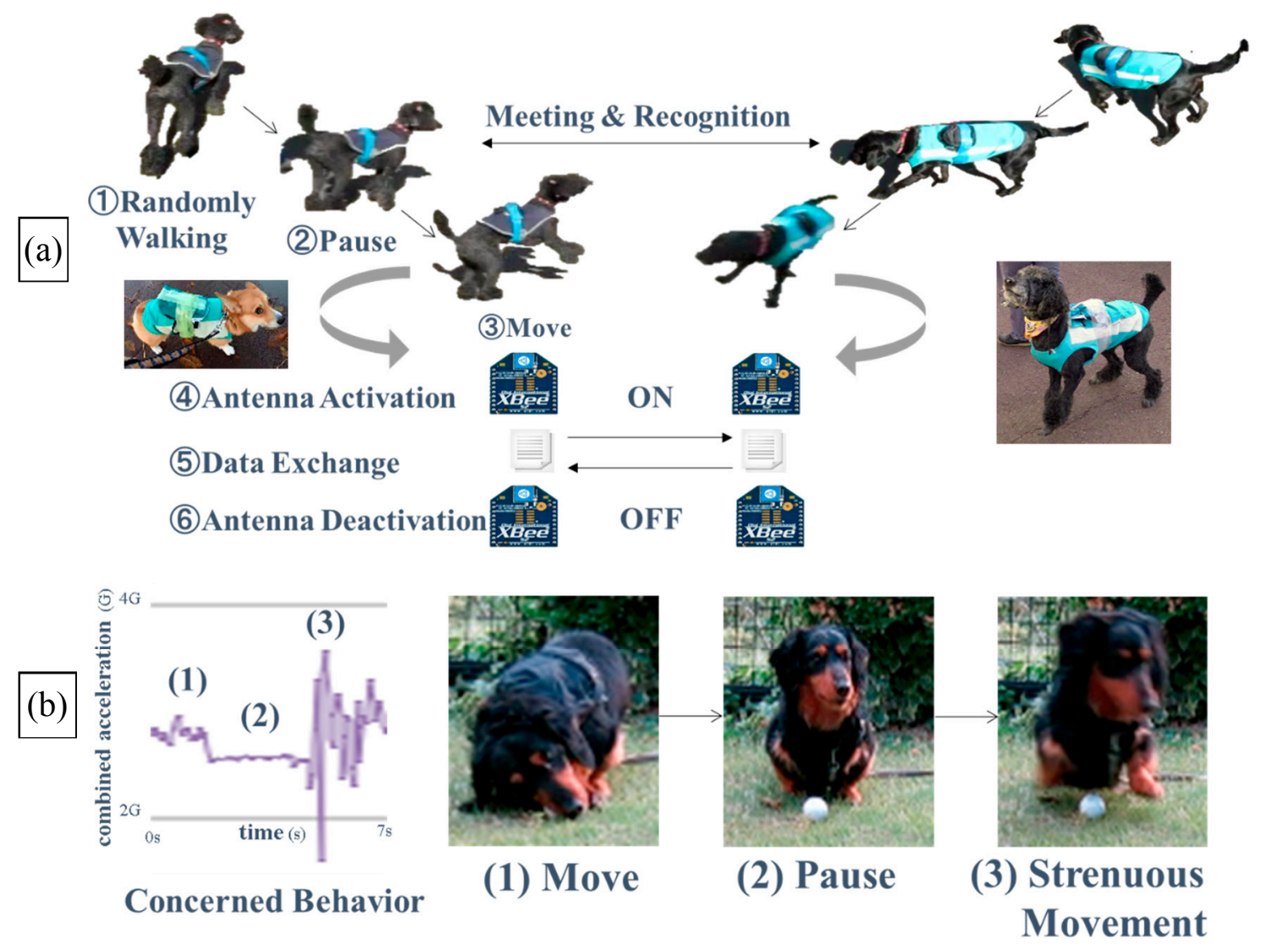

Figure 2. (a) Animal-to-animal data sharing process (1)-(6); (b) reaction state of dogs to peripheral stimuli ((1)-(3)). 
To the best of our knowledge, the proposed idea to realize significant power saving in sensor nodes by leveraging the ecological interactions of multiple wild animals is the first attempt at such an endeavor.

\section{Proposed System}

For our evaluation, we replicated the animal-to-animal data-sharing system in the Fukushima exclusion zone and observed behavior of contaminated cattle to evaluate the performance of our system in a contaminated environment. The objective of this analysis was to demonstrate that our wearable animal-to-animal network prototype (Figure 3) can function in such an environment.
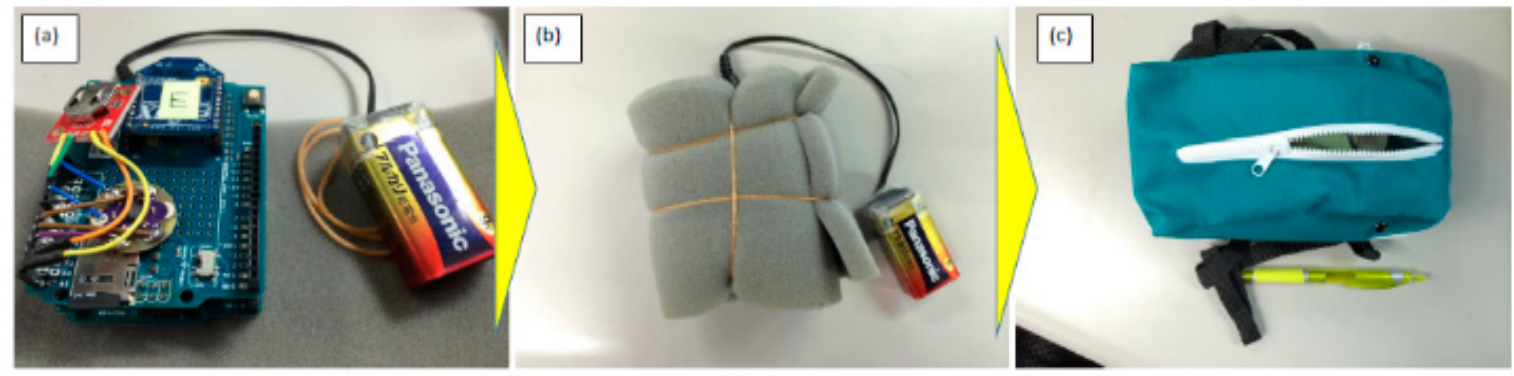

Figure 3. (a) Animal-to-animal data sharing prototype; (b) covered by sponge for preventing them from shocks; (c) put in the bag.

We used cattle in our prototype evaluation because other studies have focused on monitoring the effects of nuclear disasters on cattle [10]. We conducted our experiments by exploiting various opportunities to observe the cows exposed to radiation in the Fukushima exclusion zone, as depicted in Figures 4 and 5. We conducted our evaluation experiments by targeting two specific individuals within a herd in Figure 5a. We confirmed the following three points with residents and veterinarians who are victims of the earthquake and nuclear disasters and currently live in temporary housing $70 \mathrm{~km}$ from here. They visit this place every other day and keep feeding.

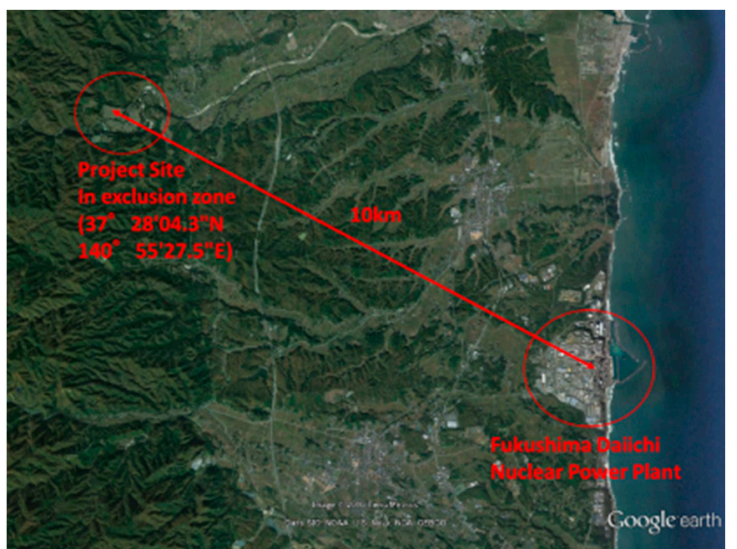

(a)

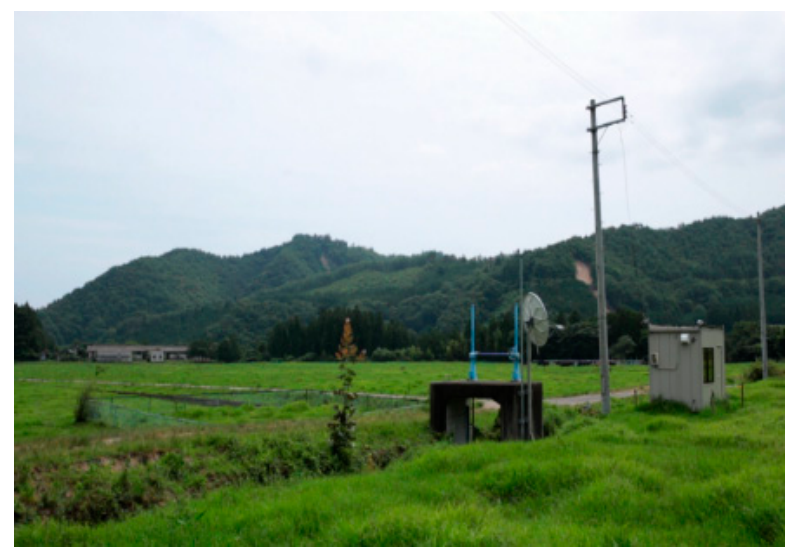

(b)

Figure 4. (a) Project site in the Fukushima exclusion zone $\left(37^{\circ} 28^{\prime} 4.3^{\prime \prime} \mathrm{N}, 140^{\circ} 55^{\prime} 27.5^{\prime \prime} \mathrm{E} ; 10 \mathrm{~km}\right.$ from Fukushima Daiichi Nuclear Power Plant) and (b) sink node station. 


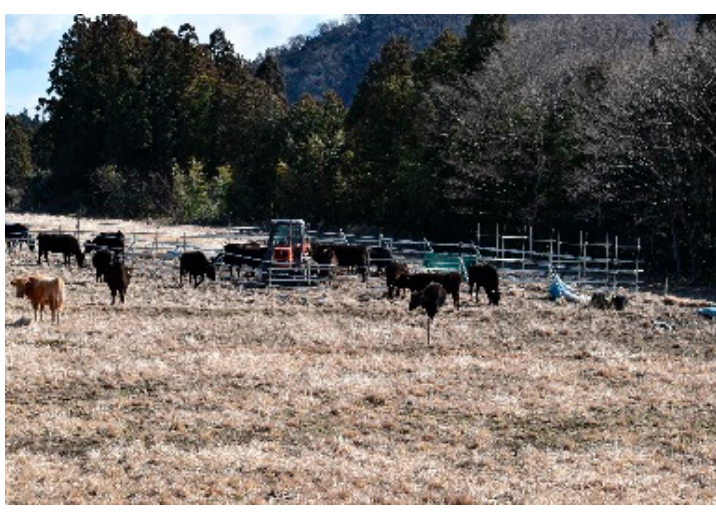

(a)

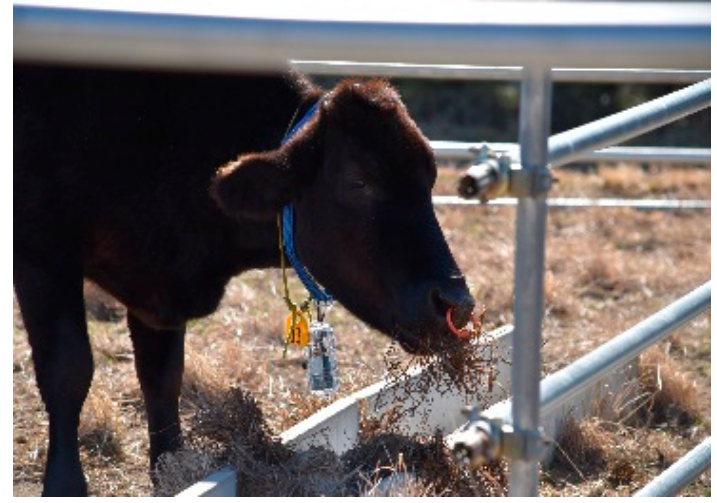

(b)

Figure 5. (a) Animals exposed to surroundings confirmed by camera at the sink node and (b) breeding cage.

1. Humanitarian priority: This is a high radiation zone; thus, it is inappropriate to bring animals into the zone for research purposes.

2. Legal compliance: All activities in this zone are restricted by the special nuclear disaster laws that were prepared after the disaster occurred. All animals in the zone were ordered killed; however, administrative agencies hesitated. As a result, the agencies could not achieve that killing; however, only surviving cattle like the ones in this herd were allowed to remain. Further, these cows are radioactive and cannot be taken out of the zone.

3. Exposure avoidance: For our research, researcher and collaborator exposure should be avoided by as much as possible. To realize the same conditions as our preliminary research, it is necessary for numerous people to induce the cows to move, which is unrealistic for the required number of people and the amount of work involved.

The data-sharing sensors in the bags shown in the Figure $3 \mathrm{c}$ were placed on the cattle chokers, as depicted in the right of Figure 5. When the cows actively encounter other cows, these sensors exchange ID information and accumulate data for each encounter. Specifically, when the sensor on Cow A sends letter " $A$ " as its ID to others, and cow B sends "B" each time they communicate. We placed a real-time clock on each sensor device and recorded the durations at which the ID data were transmitted or received. Furthermore, we can determine the number of times that the data-sharing sensors receive data. To ensure that the data are received successfully, each transmitter repeatedly sent the given letter for approximately two seconds each time a cow begins to transmit to another cow.

We used an Arduino UNO R3 with a Wireless SD Shield. On the shield, we loaded XBee ZB (Printed Circuit Board antenna type) as the ZigBee communication module, a Lilypad acceleration sensor (ADXL335), a real-time clock module, and a microSD card to record data. The sampling rate of the accelerometer was $20 \mathrm{~Hz}$, and all the mechanisms for powering the communication module were the same as those in the animal-to-animal data-sharing system. Here, communication began when displacement of the norm of the acceleration was greater than 0.2 followed by a pause of 1 to $10 \mathrm{~s}$. If 10 s have elapsed after the cow paused, the sensor resets the pause time count.

For our experiments, we used two Japanese Black cattle (Kuroge Washu), as shown in Figure 5b. Cow A was approximately eight years old, weighing approximately $400 \mathrm{~kg}$ and standing approximately at $150 \mathrm{~cm}$. Cow B was approximately three years old, weighing approximately $100 \mathrm{~kg}$ and standing approximately at $100 \mathrm{~cm}$. As the sensor weight was within the tolerable limit (i.e., no greater than $2 \%$ of the animal's body weight) [11], we assumed that the sensor weight would not impact the behavior of the cows.

We attempted to reproduce a general situation where animals freely encounter one another in a smaller area. Figure $5 b$ shows the field within the exclusion zone, including a temporary breeding 
cage. We conducted our experiment at 13:00 on 22 February 2017 for $30 \mathrm{~min}$. To prevent the cows from running away, we closed the entrance of the breeding cage. The two target cows and other cows moved freely during the experiment. In the experiments involving the cows, two specific individuals among a total of nine cows were targeted. In the wild, animals actively contact other animals for a while after their initial encounters. Therefore, we did not separate the cows. Instead, we allowed them to interact until they lost interest. Note that we continued the experiment until the two target cows could communicate successfully. In our experiments, we used JVC video cameras (Japan Victor Company, Yokohama, Japan. Gz-F200-w) to acquire the video data. We did not analyze the data at the site and did not plan re-evaluation experiment. The participants also had considerable fatigue and exceeded the allowable exposure time.

For the number of encounters (or "active" contacts), we counted the number of times a cow was observed heading toward another cow after pausing from the video data.

We observed a significant number of communication attempts in the form of visually assumable encounters or active contact. In consideration of the synchronization errors between the communication devices and video data, we allowed for a window of three seconds. We also calculated the precision, recall, and communication success rate of our data-sharing system. Regarding precision, we used the number of encounters or contact attempts as the numerator and the number of communication attempts as the denominator. For recall, we used the same numerator; however, the denominator was the specific number of encounters or contacts.

- Encounters or "active" contacts: the number of times a cow was observed heading toward another cow after pausing from the video data.

- Communication attempts: the observed number of times a significant number of communication attempts was made in the form of visually assumable encounters or active contacts.

- Communication attempts during encounters or contacts: the observed number of times a significant number of communication attempts was made during encounters or contacts.

- Communication success during encounters or contacts: the number of times a significant number of communication successes during encounters or contacts.

\section{Results}

The counts are summarized in Table 1. The encounters or "active" contacts of Cow A was 0 and that of Cow B was 1. Although we were able to visually confirm the scene encountered from the front of Cows A and B, Cow A did not visually observe a clear response to this encounter, which explains Cow A's 0 count. However, Cow B's response to this encounter was visually observable; thus, Cow B's count was 1. The numbers of communication attempts for Cows A and B were 22 and 17, respectively. It is observed that a significant number of communication attempts during the encounters or contacts. These numbers were confirmed from the log files of the attached devices. The numbers of communication attempts during encounters or contacts for Cows A and B were 21 and 17, respectively. These are the numbers of times the log file of the attached device and the video data were counted synchronously relative to the time information. The reason the number of communication attempts of Cow A during encounters or contacts is one less than the overall communication attempts is because, according to the video data, Cow A did not face Cow B in one instance. However, in this case, the attached device attempted to communicate; thus, this case was considered a false detection. According to the $\log$ files, the numbers of communication successes during encounters or contacts for Cows A and B were 10 and 10, respectively. We observed the differences between Cows A and B from these results. First, we discuss the difference between the number of communication successes. Here, each cow sometimes received the data that was transmitted by the other cow at the same time. 
Table 1. Significant counts.

\begin{tabular}{ccc}
\hline Element & Cow A & Cow B \\
\hline Encounters or "active" contacts & 0 & 1 \\
Communication attempts & 22 & 17 \\
Communication attempts during encounters or contacts & 21 & 17 \\
Communication successes during encounters or contacts & 10 & 10 \\
\hline
\end{tabular}

Based on these counts, we calculated precision, recall, and the communication success rate. Further, using the precision and recall values, we computed the F-measure (the harmonic mean of precision and recall) to measure the test's accuracy using the following equation [12].

- Precision: the number of encounters or contact attempts is the numerator; number of communication attempts is the denominator.

- Recall: the number of encounters or contact attempts is the numerator; the specific number of encounters or contacts is the denominator.

$$
F=\frac{\left(1+\beta^{2}\right) \times \text { precision } \times \text { recall }}{\left(\beta^{2} \times \text { precision }\right)+\text { recall }}
$$

To evaluate the precision and recall equally, we adopted $\beta=1$ [6]. We calculated the ratio of time the communication module did not consume power versus the duration of the experiment (i.e., the time-saving rate of communication).

The results are depicted in Figure 6. The precision, recall, F-measure, and communication success rate values were $95.42 \%, \mathrm{~N} / \mathrm{A}, \mathrm{N} / \mathrm{A}$, and $47.62 \%$ for Cow A, respectively, and $100 \%, 1700 \%, 189 \%$, and $58.82 \%$ for Cow B, respectively. The recall of Cow A is N/A because the denominator (encounters or "active" contacts) is zero, and the F-measure of Cow A is also N/A because the numerator value (i.e., recall) is N/A. We could calculate the recall and F-measure of cow A if the value of encounters or "active" contacts of Cow A was greater than or equal to 1; however, we confirmed the visual data of Cow A over and over.

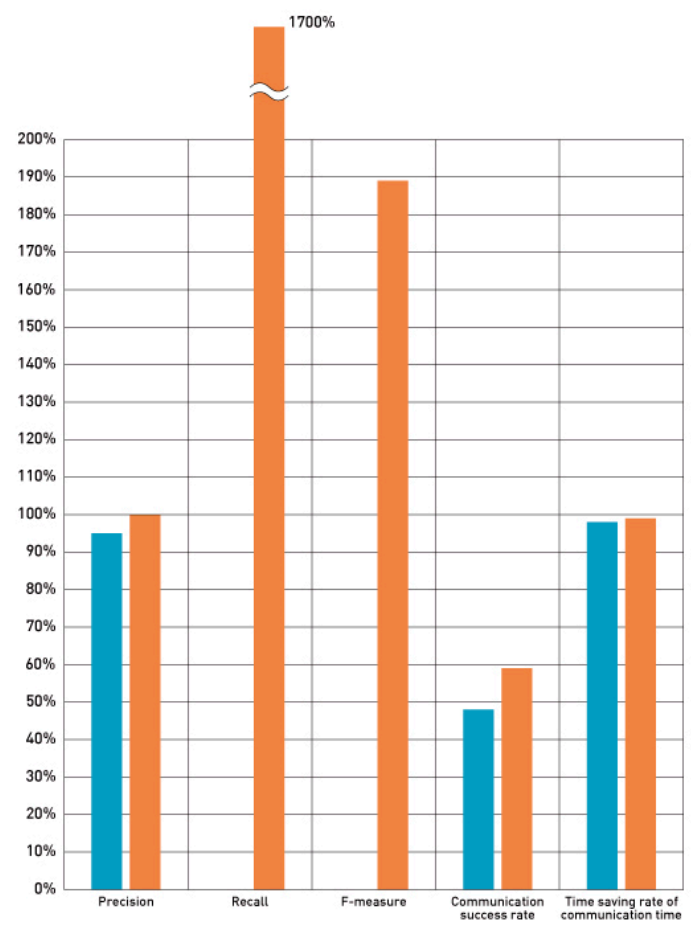

Figure 6. Results of animal-to-animal data sharing experiment between Cows A and B (among nine total cows) within the Fukushima exclusion zone. 
The results indicate that Cow B could communicate with Cow A accurately at approximately $40 \%$ to $60 \%$ based on the recall value of $1700 \%$ and an F-measure value of $189 \%$ (for Cow B). However, since the F-measure is a ratio, $1700 \%$ and $189 \%$ are abnormal values.

Note that it is difficult to conduct additional experiments due to the exposure problem. Therefore, the results are described in Figure 7 in reference to the results of a previous research [6]. Due to the numerical values that could not be calculated and the abnormal values, it is impossible to accurately evaluate this system. However, as the communication is successful, it is possible to estimate the situation relative to the reason due to which the numerical values and abnormal values occurred. Therefore, the factors that resulted in the number of communication trials (i.e., 22) when the number of measured encounters was zero can summarized as follows. (1) Although there was an encounter between Cows A and B, it could not be confirmed visually. The visible reaction is difficult to understand as compared to that of a dog; (2) Although there was no encounter between Cows A and B, there was a chance that communicating occurred when Cows A and B herd of cattle encountered the seven other cows.

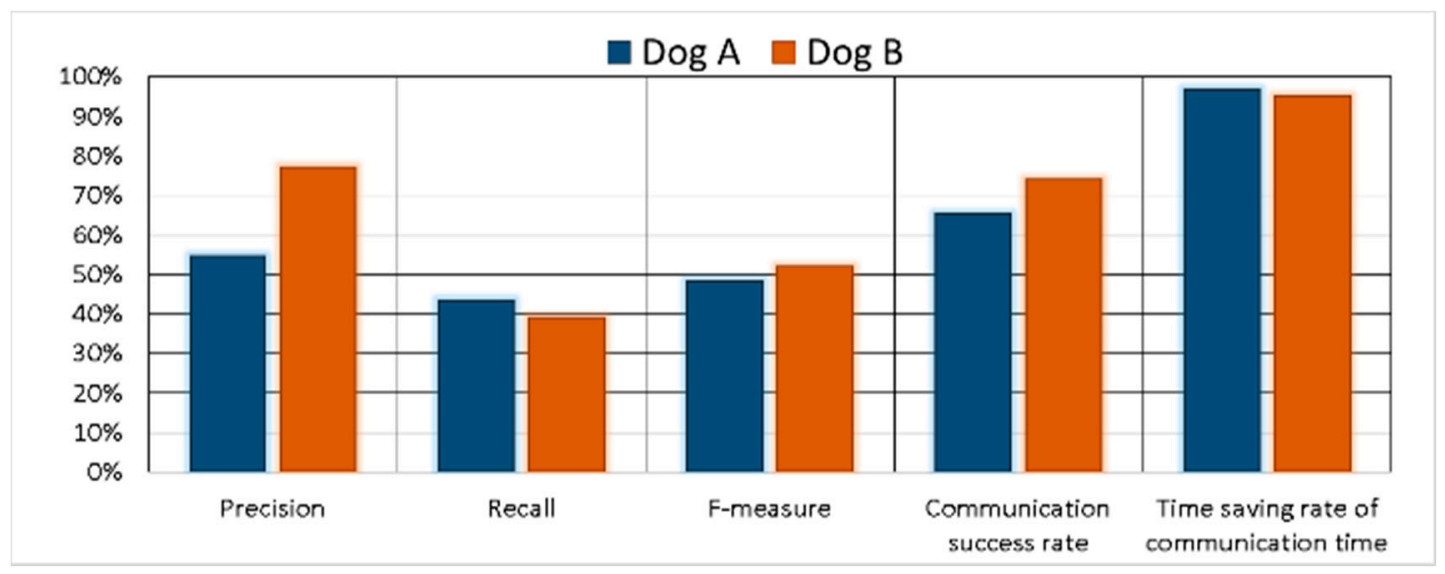

Figure 7. Results of animal-to-animal data sharing experiment between two laboratory dogs.

In the experiments involving the cows, two specific individuals. The two individuals are in a herd of nine cows.

Finally, as compared to the conventional method, we observed that the communication power consumption was reduced by $98.52 \%$ for Cow A and $98.58 \%$ for Cow B. This indicates that the communication devices were powered off more than $95 \%$ of the time during the 30 -min experiment. In the conventional method, the presence of adjacent sensor nodes is detected using radio waves. Therefore, with the conventional method, it is necessary to keep the communication devices powered up for the duration of the experiment. In contrast, our method realizes effective and substantial power-saving performance while preserving the weight limitation of animal-mounted sensors (less than $2 \%$ ).

In our experiments, we were able to construct some index scales of concrete individual differences between the animals relative to the differences in the precision and recall values of the communication attempts during encounters or contacts.

\section{Discussion}

Although we could experimentally confirm successful operation of the proposed method using animals inhabiting the Fukushima exclusion zone, a comparative evaluation with the previous experiment was not performed. It is difficult to perform reevaluation experiments due to the exposure problem. Therefore, we will clarify the cause of the problem by comparing the results of preliminary research and discuss future considerations. As stated previously, the numerical values that could not be calculated and the abnormal values made it impossible to accurately evaluate the proposed system. 
However, communication between Cows A and B succeeded; therefore, it is possible to estimate the situation from the reason why the numerical values and abnormal values occurred. Therefore, we hypothesize that the factors that resulted in the number of communication trials (22) when the number of measured encounters was zero were the (1) visually unconfirmable reactions and (2) interaction with other individuals.

1. Although there was an encounter between Cows A and B, it could not be confirmed visually. The visible reaction of Cow A was difficult to understand as compared to that of Cow B.

2. Although there was no encounter between Cows $A$ and $B$, there was a chance that the communicating occurred when Cows A and B encountered the seven other cows.

Problems that cannot be computed due to the visually unconfirmable reactions are affected by the design of the algorithm. The protocol of this system is designed based on a dog's response as observed by the human eye, as depicted in Figure 2b. In other words, if there is insufficient response as observed by a human or if there is little response, an appropriate evaluation cannot be performed. However, the numerical value of communication attempts as measured by the device was greater than or equal to 1 . Even in the case of Cow A, which demonstrated poor visible reaction, it is possible that the device itself measured the reaction. Obviously, false positives are possible; however, such cases can be considered hints while developing new algorithms if they are considered to be the behavior of animals.

The false positive may have been caused by the design of the algorithm caused by encounters with other individuals. This algorithm was developed assuming a situation where two individuals, such as those in Figure 2a, encounter each other. Specifically, the conditions of this protocol differ, i.e., it measures one-on-one dogs under controlled conditions (people-induced inquiries). In the experiments involving the cows, two individuals among a total of nine cows were targeted, and the experiments were conducted under semi-controlled conditions (release). Although misdetection is possible, this is useful knowledge to gain, relative to the development of new algorithms in which the device considers animal behavior.

\subsection{Coverage Problem}

As discussed previously, animal-to-animal data sharing is a system in which wild animals wear sensors that record spatial information (e.g., GPS, radiation dose, and bioacoustics) within their territory. Further, the obtained information is shared through group actions and is eventually uploaded to the Internet. Furthermore, this procedure occurs with substantially reduced power requirements.

Our objective was to address the aforementioned coverage, operational time, and reliability problems. Therefore, we conclude that our animal-to-animal data-sharing system realizes a proof of concept, and we have a system that can be extended to animals for which there is no possibility of recapture.

Furthermore, our dog and cow experiments have demonstrated that animal-to-animal network systems work for such animals; however, the algorithms we used depend on the relative level of the reactions of the animals. When targeting an animal that acts independently, such as a dog, communication at encounter is considered to be intended by the algorithm; however, while targeting animals, such as cows, communication with other individuals in the herd occurs. This is unintended by the algorithm, and as a result it causes false positives and unintended communications. In future, it will be necessary to conduct experiments to establish communication between animals that can crowd and those that do not.

\subsection{Application}

Following the Chernobyl incident, remediation aimed to improve the radiological conditions for plants and animals in the exclusion zone that would further exhibited adverse impacts on the biosphere [13]. The recovery of the affected biosphere in the Chernobyl exclusion zone has been confounded by an overriding response to the elimination of human activities (e.g., termination of 
agricultural and industrial activities and the accompanying environmental pollution in the affected area). Ideally, recovery efforts within affected areas would have a neutral effect on these remote and contaminated landscapes. The Fukushima exclusion zone is the most radioactively polluted zone in the Fukushima area. No remote sensing method at the surface level is available due to a lack of power and information infrastructures. Field surveys with strict identity checks are required to enter the zone, and workable hours are extremely limited due to radiation exposure concerns. Portable recorders used in soundscape studies require regular replacement of memory devices and batteries, which is impractical for long-term and continuous investigation in the exclusion zone [14]. This study has attempted to provide wearable microphone sensors for wildlife with an animal-to-animal data sharing mechanism as we move toward a sustainable worldwide society.

\section{Conclusions}

We have developed a model to evaluate animal-to-animal data sharing in a remote environment and implemented animal behavior-oriented triggers to elicit data exchange between animals. Furthermore, our dog (previous study) and cow (current study) experiments demonstrated that animal-to-animal network systems were functional for such animals; however, the algorithms that were used were dependent on the relative level of the reactions of the animals. While targeting an independent animal, such as a dog, communication at encounter can be achieved, as intended by the algorithm; however, while targeting animals, such as cows, communication with other individuals in the herd occurred. This study has provided a conceptual overview of animal-to-animal interaction, discussed animal-wearable sensors for monitoring animal territories and animal-to-animal data sharing, and presented an evaluation of an implementation within the Fukushima exclusion zone.

Author Contributions: Conceptualization, H.H.K.; Funding acquisition, H.H.K., H.K.; Methodology, K.N., K.M., Y.S. and K.S.; Project administration, H.H.K.; Supervision, H.H.K.; Writing-original draft, H.H.K.; Writing-review \& editing, B.N.

Funding: This study was supported by JSPS KAKENHI Grants 26700015 and 16K12666, MIC SCOPE Grants 142103015 and 162103107, JST PRESTO 11012, the Telecommunications Advancement Foundation, the Moritani Scholarship Foundation, the Tateisi Science and Technology Foundation, and the Mitsubishi Foundation.

Acknowledgments: Special thanks go to Fumikazu Watanabe and Laboratory Members.

Conflicts of Interest: The authors declare no conflict of interest.

\section{References}

1. Doniec, M.; Detweiler, C.; Vasilescu, I.; Anderson, D.M.; Rus, D. Autonomous gathering of livestock using a multi-functional sensor network platform. In Proceedings of the 6th Workshop on Hot Topics in Embedded Networked Sensors, Killarney, Ireland, 28-29 June 2010; ACM: New York, NY, USA, 2010; pp. 1-5.

2. Pollard, J.K.; Rohman, S.; Fry, M.E. A Web-based mobile medical monitoring system. In Proceedings of the International Workshop on Intelligent Data Acquisition and Advanced Computing Systems: Technology and Applications, Crimea, Ukraine, 4 July 2001; pp. 32-35.

3. Small, C. Estimation of urban vegetation abundance by spectral mixture analysis. Int. J. Remote Sens. 2001, 22, 1305-1334. [CrossRef]

4. Nakagawa, K.; Kobayashi, H.; Sezaki, K. Carrier pigeon-like sensing system: Animal-computer interface design for opportunistic data exchange interaction for a wildlife monitoring application. In Proceedings of the 5th Augmented Human International Conference, Kobe, Japan, 7-9 March 2014; ACM: Kobe, Japan, 2014; pp. 1-2.

5. Kobayashi, H.; Ueoka, R.; Hirose, M. Human computer biosphere interaction: Towards a sustainable society. In CHI '09 Extended Abstracts on Human Factors in Computing Systems; ACM: Boston, MA, USA, 2009; pp. 2509-2518.

6. Kobayashi, H. Basic Research in Human-Computer-Biosphere Interaction. Ph.D. Thesis, The University of Tokyo, Tokyo, Japan, 2010. 
7. Ogura, J. Japanese Scientists to Use Wild Monkeys to Track Radiation. Available online: http:/ / edition.cnn. com/2011/12/14/world/asia/japan-nuclear-monkeys/index.html (accessed on 20 April 2018).

8. Begon, M.; Harper, J.L.; Townsend, C.R. Ecology: Individuals, Populations, and Communities, 2nd ed.; Blackwell Scientific: Boston, MA, USA, 1990.

9. Hewett; Baecker; Card; Carey; Gasen; Mantei; Perlman; Strong; Verplank. Definition of Human Computer Interaction. Available online: http://old.sigchi.org/cdg/cdg2.html (accessed on 20 April 2018).

10. Okada, K.; Sato, I.; Deguchi, Y.; Morita, S.; Yasue, T.; Yayota, M.; Takeda, K.; Sato, S. Distribution of radioactive cesium in edible parts of cattle. Anim. Sci. J. 2013, 84, 798-801. [CrossRef] [PubMed]

11. Kobayashi, H.; Hiyama, A.; Kobayashi, S.; Izawa, M.; Matsushima, J.; Michitaka, H. Wild Theremin: Electronic music instrument for remote wildlife observation. Trans. Hum. Interface Soc. 2010, 12, 15-22.

12. Hripcsak, G.; Rothschild, A.S. Agreement, the F-Measure, and Reliability in Information Retrieval. J. Am. Med. Inf. Assoc. 2005, 12, 296-298. [CrossRef] [PubMed]

13. Chernobyl Forum; Expert Group Environment; International Atomic Energy Agency. Environmental Consequences of the Chernobyl Accident and Their Remediation: Twenty Years of Experience; Report of the Chernobyl Forum Expert Group 'Environment'; International Atomic Energy Agency: Vienna, Austria, 2006; p. 137.

14. Ishida, K. Contamination of Wild Animals: Effects on Wildlife in High Radioactivity Areas of the Agricultural and Forest Landscape. In Agricultural Implications of the Fukushima Nuclear Accident; Nakanishi, T.M., Tanoi, K., Eds.; Springer: Tokyo, Japan, 2013; pp. 119-129.

(C) 2018 by the authors. Licensee MDPI, Basel, Switzerland. This article is an open access article distributed under the terms and conditions of the Creative Commons Attribution (CC BY) license (http://creativecommons.org/licenses/by/4.0/). 